\title{
Gauged supersymmetric $\sigma$-models and soft breaking terms
}

\author{
T.S. Nyaweld* \\ The Abdus Salam ICTP, Strada Costiera 11, I-34014 Trieste, Italy.
}

\begin{abstract}
Supersymmetric non-linear $\sigma$-models in four dimensions with $D$-term potentials can sometimes have a singular metric. As the kinetic terms of scalar fields and their chiral fermionic partners are determined by this metric, it follows that their kinetic energy vanishes in the vacuum. In previous work we have shown for a simple model that this degeneracy of the sigma-model metric can be lifted by soft supersymmetry breaking terms. In this letter I introduce soft breaking terms in more realistic models based on $S O(10)$ and $E_{6}$ and compute their resulting mass spectra.
\end{abstract}

PACS numbers: $11.30 . \mathrm{Bb}, 12.60 . \mathrm{Jv}$

The kinetic part of a lagrangian for chiral multiplets in supersymmetric sigma-models in four dimensions is determined by the metric of the Kähler manifold [1, 2]. However, it is not automatically guaranteed that this metric is always invertible. In previous work [3], it was found that the Kähler metric develops zero-modes in the vacuum: the metric for the Goldstone bosons and their fermionic partners vanishes. In [4] we have shown for a simple model that soft supersymmetry breaking terms can shift the minimum away from the singular point and provide a consistent mass-spectrum. In this letter, I introduce soft supersymmetry breaking terms in more realistic models based on the coset spaces $E_{6} /[S O(10) \times U(1)]$ and $S O(10) / U(5)$.

From the point of view of unification the coset space $S O(10) /[S U(5) \times U(1)]$ is a very interesting for phenomenological applications as both $S O(10)$ and $S U(5)$ are often used GUT groups [5]. Since supersymmetric pure $\sigma$-models on coset spaces $G / H$ of Kähler type, are known to be anomalous 6, 7, 8, 9], a supersymmetric model built on the $S O(10) /[S U(5) \times U(1)]$ is not free of anomalies by itself as all the $\underline{10}$ anti-symmetric complex coordinates $z^{i j}$ and their chiral superpartners $\psi_{L}^{i j}(i, j=1, \ldots, 5)$ of this manifold carry the same charges. To construct a consistent supersymmetric model on this coset one has to include the fermionic partners of the coordinates in an anomaly-free representation. As $S U(5)$ representations are not anomaly free by themselves, we have to use the full $S O(10)$ representations for our additional matter coupling in this case. This has been achieved in [3] by introducing a singlet $\underline{1}$ and a completely anti-symmetric tensor with 4 indices, which is equivalent to $\underline{\overline{5}}$ to complete the set of complex chiral superfields to form a $\underline{16}$ of $S O(10)$. The antisymmetric coordinates of the coset are combined into a $\underline{10}$ of $S U(5)$ with a unit $U(1)$ charge. An anomaly free representation is obtained using the branching of the $\underline{16}$. Indeed, its decomposition under $S U(5)$ reads: $\underline{16}=\underline{10}(1)+\underline{\overline{5}}(-3)+\underline{1}(5)$, where the numbers in parentheses denote the relative $U(1)$ charges. Therefore, the

*tnyawelo@ictp.trieste.it. supersymmetric model on $S O(10) / U(5)$ is defined by three chiral superfields $\left(\Phi^{i j}, \Psi_{i}, \Psi\right)$ : the target manifold $S O(10) / U(5)$ is parametrized by 10 anti-symmetric complex fields $z^{i j}$ in a chiral superfield $\Phi^{i j}=\left(z^{i j}, \psi_{L}^{i j}, H^{i j}\right)$, to which are added $S U(5)$ vector and scalar matter multiplets denoted respectively by $\Psi_{i}=\left(k_{i}, \omega_{L}, B_{i}\right)$, and $\Psi=\left(h, \varphi_{L}, F\right)$.

The complete Kähler potential of the model is obtained using the method developed in 10, 11]:

$$
\begin{aligned}
\mathcal{K}(z, \bar{z} ; k, \bar{k} ; h, \bar{h})= & \frac{1}{2 f^{2}} \ln \operatorname{det} \chi^{-1}+(\operatorname{det} \chi)^{2}|h|^{2} \\
& +(\operatorname{det} \chi)^{-1} k \chi^{-1} \bar{k}
\end{aligned}
$$

with the submetric $\chi^{-1}=\mathbb{1}_{5}+f^{2} z \bar{z}$. The dimensionful constant $f$ is introduced to assign correct physical dimensions to the scalar fields $(z, \bar{z})$. The Kähler metric derived from this Kähler potential $\mathcal{K}$ possesses a set of holomorphic Killing vectors generating a non-linear representation of $S O(10)$ :

$$
\begin{aligned}
\delta z & =\frac{1}{f} x-u^{T} z-z u+f z x^{\dagger} z, \\
\delta h & =2 \operatorname{tr}\left(f z x^{\dagger}-u^{T}\right) h, \\
\delta k & =-k\left(-u^{T}+f z x^{\dagger}+\operatorname{tr}\left(-u^{T}+f z x^{\dagger}\right) \mathbb{1}_{5}\right) .
\end{aligned}
$$

Here $u$ represents the parameters of the linear diagonal $U(5)$ transformations, and $\left(x, x^{\dagger}\right)$ are the complex parameters of the broken off-diagonal $S O(10)$ transformations.

In order for the chiral fermions $\left(\psi_{L}^{i j}, \omega_{L i}, \varphi_{L}\right)$ to have a physical interpretation as describing a family quarks and leptons, gauge interactions should be introduced. In this case supersymmetry implies the addition of a potential from elimination of the auxiliary $D^{i}$ fields by substitution for the Killing potentials [17, 18. We will consider two different options: gauging of the full $S O(10)$ symmetry, and gauging of the stability subgroup $S U(5) \times U(1)$ only. We denote collectively the $S O(10)$ gauge fields as $A_{\mu}=\left(U_{\mu}, W_{\mu}^{\dagger}, W_{\mu}\right)$ with $W_{\mu}^{\dagger}$ and $W_{\mu}$ the gauge fields corresponding to the broken $S O(10)$ transformations parametrized by $\left(x, x^{\dagger}\right)$ and with $U_{\mu}$, the gauge field 
of the diagonal transformations parametrized by $u$. This requires the introduction of covariant derivatives for the dynamical fields. We give here the covariant derivatives for the scalar fields only:

$$
\begin{aligned}
D_{\mu} z= & \partial_{\mu} z-g_{10}\left(\frac{1}{f} W_{\mu}-U_{\mu}^{T} z-z U_{\mu}+f z W_{\mu}^{\dagger} z\right), \\
D_{\mu} k= & \partial_{\mu} k+g_{10} k\left(f W_{\mu}^{\dagger} z-U_{\mu}^{T}\right. \\
& \left.\quad+\operatorname{tr}\left(f W_{\mu}^{\dagger} z-U_{\mu}^{T}\right) \mathbb{1}_{5}\right), \\
D_{\mu} h= & \partial_{\mu} h-2 g_{10} \operatorname{tr}\left(f W_{\mu}^{\dagger} z-U_{\mu}^{T}\right) h .
\end{aligned}
$$

For the $D$-term scalar potential we need the $S O(10)$ Killing potentials. The full Killing potential $\mathcal{M}$ generating the Killing vectors (2) can be written as

$$
\mathcal{M}\left(u, x^{\dagger}, x\right)=\operatorname{tr}\left(u \mathcal{M}_{u}+x^{\dagger} \mathcal{M}_{x}+x \mathcal{M}_{x}^{\dagger}\right)
$$

where $\mathcal{M}_{u}$ is the $U(5)$ Killing potentials, and $\left(\mathcal{M}_{x}, \mathcal{M}_{x^{\dagger}}\right)$ are the broken Killing potentials. The $U(1)$ Killing potential $\mathcal{M}_{Y}$ is defined as the trace of $U(5)$ Killing potential $\mathcal{M}_{u}$ whereas the remaining $S U(5)$ Killing potential $\mathcal{M}_{t}$ is defined as a traceless part of $\mathcal{M}_{u}$ :

$$
\mathcal{M}_{t}=\mathcal{M}_{u}-\frac{1}{5} \mathcal{M}_{Y} \mathbb{1}_{5}, \quad \mathcal{M}_{Y}=\operatorname{tr} \mathcal{M}_{u}
$$

The coupling of the gauge multiplets to the supersymmetric non-linear $\sigma$-model on $S O(10) / U(5)$ has interesting consequences for the spectrum. It can induce spontaneous breaking of supersymmetry, and further spontaneous breaking of the internal symmetry. For example, if we gauge the full $S O(10)$, all the Goldstone bosons $(z, \bar{z})$ are absorbed by the vector bosons $\left(W_{\mu}^{\dagger}, W_{\mu}\right)$ which become massive. In this case we may choose to study the model in the unitary gauge $z=\bar{z}=0$. However, it was found in [3] that in this gauge, the Kähler metric develops zero-modes in the vacuum: the metric $G_{\sigma z^{i j} \bar{z}_{k l}}$ for the Goldstone bosons and their fermions vanishes.

To see this, we start from the scalar potential. As already stated, we choose the unitary gauge: $z=\bar{z}=0$. Then the potential ${ }^{1}$ for the fully gauged $S O(10)$ model reads

$$
V_{\text {unitary }}=\frac{g_{10}^{2}}{10}\left(10|h|^{2}-\frac{5}{2 f^{2}}-6|k|^{2}\right)^{2}+\frac{2}{5} g_{10}^{2}\left(|k|^{2}\right)^{2}(6)
$$

From this we see that we only have a supersymmetric minimum if

$$
|k|^{2}=0, \quad|h|^{2}=\frac{1}{4 f^{2}}
$$

\footnotetext{
${ }^{1}$ With the given field content, it is not possible to construct an $S O(10)$-invariant trilinear superpotential. Thus the full potential contains only a $D$-term potential.
}

It can be seen immediately that this solution yields the vanishing of the Kähler metric:

$$
\begin{aligned}
\left\langle G_{\sigma(i j)}{ }^{(k l)}\right\rangle= & \delta_{i}^{[k} \delta_{j}^{l]}\left(\frac{1}{2 f^{2}}-2|h|^{2}+\left|k_{i}\right|^{2}\right) \\
& +k^{(k} \delta_{(i}^{l)} \bar{k}_{j)}=0 .
\end{aligned}
$$

In this case the kinetic terms of the Goldstone superfield components vanish, therefore, mass terms for the $S O(10)$ gauge fields $\left(W_{\mu}^{\dagger}, W_{\mu}\right)$ vanish as well. Moreover, the theory becomes strongly coupled, with some of the four-fermion interactions exploding, namely:

$$
\mathcal{L}_{4-\text { ferm }}=R_{z \bar{z} h \bar{h}} \bar{\psi}_{R} \psi_{L} \bar{\varphi}_{L} \varphi_{R}+\text { perm }
$$

with the curvature components given by

$$
\begin{aligned}
\left\langle R_{z \bar{z} h \bar{h}}\right\rangle & =\left\langle R_{(i j)}^{(k l)}{ }_{h \bar{h}}\right\rangle \\
& =-2 f^{2} \delta_{i}^{[k} \delta_{j}^{l]}\left(1+2|h|^{2}\left(\frac{1}{2 f^{2}}-2|h|^{2}\right)^{-1}\right) .
\end{aligned}
$$

This may point to a restauration of the $S O(10)$ symmetry. However, not all of the physics behind this model is as yet understood.

To avoid the problem of vanishing of the Kähler metric, we shift the minimum of the potential away from the singular point by adding $S O(10)$-invariant soft supersymmetry breaking scalars mass terms

$$
\Delta V=\mu_{1}^{2}(\operatorname{det} \chi)^{2}|h|^{2}+\mu_{2}^{2}(\operatorname{det} \chi)^{-1} k \chi^{-1} \bar{k}
$$

to the potential. As a result the minimum of the potential is shifted to a position where the expectation value of the Kähler metric is not vanishing; and the scalar $h$ gets a vacuum expectation value

$$
|k|^{2}=0, \quad|h|^{2}=v^{2}=\frac{1}{4 f^{2}}-\frac{\mu_{1}^{2}}{20 g_{10}^{2}}, \quad \mu_{1}^{2}<\frac{5 g_{10}^{2}}{f^{2}},
$$

breaking the linear local $U(1)$ subgroup. The corresponding $U(1)$ vector becomes massive; and the remaining vectors of $S U(5)$ stay massless. In the fermionic sector, two Dirac fermions are realized as a combination of the fermions of the chiral multiplets with the gauginos.

We now present details of the above mass spectrum. Since in general $S O(10)$ is broken in the vacuum, the Goldstone bosons $(\bar{z}, z)$ are absorbed in the longitudinal component of the charged vector bosons, and we may choose the unitary gauge $\bar{z}=z=0$. Before computing the mass spectrum of the theory, we first decompose the $U(5)$ vector multiplet $U_{j}^{i}=\left(U_{\mu j}^{i}, \Lambda_{R j}^{i}\right)$ into a $U(1)$ and $S U(5)$ vector multiplets denoted respectively by $A=\left(A_{\mu}, \lambda_{R}\right)$ and $V_{j}^{i}=\left(V_{\mu j}^{i}, \lambda_{R j}^{i}\right)$ :

$$
V=U-\frac{1}{5} A \mathbb{1}_{5} \quad \operatorname{tr}(V)=0, \quad A=\operatorname{tr}(U) .
$$

It follows that the kinetic terms for the $U(1)$ multiplet are not canonically normalized. To obtain the standard 
normalization, we redefine the $U(1)$ multiplet according to

$$
A \rightarrow \sqrt{5}\left(\tilde{A}_{\mu}, \tilde{\lambda}_{R}\right)
$$

With the redefined fields, the part of the lagrangian that determine the mass spectrum of the theory; in the unitary gauge $z=\bar{z}=0$ reads:

$$
\begin{aligned}
\mathcal{L}= & -\delta_{i}^{[k} \delta_{j}^{l]} \frac{\mu_{1}^{2}}{10 g_{10}^{2}}\left(g_{10}^{2} W^{\dagger(i j)} \cdot W_{(k l)}+\bar{\psi}_{L}^{(i j)} \stackrel{\leftrightarrow}{\partial} \psi_{(k l) L}\right) \\
& -\left(\frac{1}{2}(\partial \rho)^{2}+20 g_{10}^{2} v^{2} \rho^{2}+20 g_{10}^{2} v^{2} \tilde{A}^{2}+\bar{\varphi}_{L} \stackrel{\leftrightarrow}{\partial} \varphi_{L}\right) \\
& -\left(\frac{1}{2} \partial \tilde{k} \cdot \partial \tilde{k}+\frac{1}{2 f^{2}}\left(\frac{3 \mu_{1}^{2}}{5}+\mu_{2}^{2}\right) \tilde{k}^{2}+\bar{\omega}_{L}^{i} \stackrel{\leftrightarrow}{\partial} \omega_{i L}\right) \\
& -\frac{1}{4}\left[\frac{1}{2} \bar{F}_{(i j)}(W) \cdot F^{(i j)}(W)+F_{\mu \nu}^{2}(\tilde{A})\right. \\
& \left.+F^{i}{ }_{j}(V) \cdot F^{i}{ }_{j}(V)\right]-\tilde{\bar{\lambda}}_{R} \stackrel{\leftrightarrow}{\partial} \tilde{\lambda}_{R}-\bar{\lambda}_{R j}^{i} \stackrel{\leftrightarrow}{\partial} \lambda_{R}^{i} j \\
& -\frac{1}{2}\left(\frac{1}{2} \bar{\lambda}_{R}^{(i j)} \stackrel{\leftrightarrow}{\partial}_{\lambda} \lambda_{(i j) R}+\frac{1}{2} \bar{\lambda}_{L}^{(i j)} \overleftrightarrow{\not \partial}_{\left.\lambda_{(i j) L}\right)}\right) \\
& +2 \sqrt{2} g_{10} G_{\sigma(i j)}(k l)\left[\frac{1}{f} \bar{\lambda}_{R}^{(i j)} \psi_{L(k l)}+\text { h.c. }\right] \\
& +2 \sqrt{2} g_{10}\left[2 \sqrt{5} v \tilde{\bar{\lambda}}_{R} \varphi_{L}+\text { h.c. }\right] .
\end{aligned}
$$

In this expression we have expand the full potential (6) and (11) to second order in fluctuations $\rho$ and $\tilde{k}$ with scalar $\rho$ defined by $h=\left(v+\frac{1}{\sqrt{2}} \rho\right) e^{\frac{1}{\sqrt{2} v} i \alpha}$ around the absolute minimum (12). The bosonic mass spectrum can be read off easily form the lagrangian (15); they read:

$$
\begin{aligned}
\mathrm{m}_{W}^{2} & =\frac{4}{f^{2}} \frac{\mu_{1}^{2}}{10}, \quad \mathrm{~m}_{\rho}^{2}=\mathrm{m}_{\tilde{A}}^{2}=40 g_{10}^{2} v^{2}, \\
\mathrm{~m}_{\tilde{\kappa}}^{2} & =\frac{1}{f^{2}}\left(\frac{3 \mu_{1}^{2}}{5}+\mu_{2}^{2}\right) .
\end{aligned}
$$

In the fermionic sector, two Dirac fermions are formed by combining the quasi-Goldstone fermions $\psi_{L}^{[i j]}$ and $\varphi_{L}$ with the right-handed gauginos $\lambda_{R}^{[i j]}$ and $\tilde{\lambda}_{R}$

$$
\Psi=\tilde{\lambda}_{R}+\varphi_{L}, \quad \Lambda^{[i j]}=\frac{\mu_{1}}{g_{10} \sqrt{10}} \psi_{L}^{[i j]}+\frac{1}{2} \lambda_{R}^{[i j]},
$$

with masses: $\mathrm{m}_{\Psi}=2 g_{10} v \sqrt{10}$ and $\mathrm{m}_{\Lambda}=\frac{\sqrt{2} \mu_{1}}{\sqrt{5} f}$. The $\underline{\overline{5}}$ of the left-handed chiral fermions $\omega_{i L}$, the $\underline{10}$ of the left-handed gaugino's $\lambda_{L}^{[i j]}$, and the Majorana fermions $\lambda_{R j}^{i}$ that are the gauginos of the unbroken $S U(5)$ symmetry remain massless. Notice here that in the limit $\mu_{1,2}^{2} \rightarrow 0$ and $g_{10}=g_{1}$, one gets the same massive multiplets in the model with only gauged linear subgroup $S U(5) \times U(1)$. The only difference is that in the case of gauged linear subgroup $S U(5) \times U(1)$ there are 20 massless Goldstone bosons $(\tilde{\bar{z}}, \tilde{z})$, and their superpartners $\left(\psi_{L}, \bar{\psi}_{L}\right)$; and no gauge bosons $(\bar{W}, W)$ of the 20 broken generators of $S O(10)$. (We have observed a similar thing to happen also in $E_{6} / S O(10) \times U(1)$ model discussed below.)

As an alternative to gauging $S O(10)$, one can gauge only the linear subgroup $S U(5) \times U(1)$ instead. It is then allowed in this case to introduce a Fayet-Iliopoulos term with parameter $\xi$. It turns out that the corresponding models are indeed well-behaved ${ }^{2}$ for a range of non-zero values of this parameter as show below.

To determine the physical realization and the spectrum of the theory, we have to minimize the potential

$$
V=\frac{g_{1}^{2}}{10}\left(\xi-i \mathcal{M}_{Y}\right)^{2}+\frac{g_{5}^{2}}{2} \operatorname{tr}\left(-i \mathcal{M}_{t}\right)^{2} .
$$

This potential has absolute minimum at zero if

$$
\begin{aligned}
& |z|^{2}=|k|^{2}=0, \quad-\frac{5}{2 f^{2}} \leq \xi<0, \\
& |h|^{2}=\frac{1}{4 f^{2}}+\frac{1}{10} \xi=v^{2} .
\end{aligned}
$$

This solution is supersymmetric and spontaneously breaks $U(1)$, whilst $S U(5)$ is manifestly preserved. As a result, the $U(1)$ gauge field $\tilde{A}$ become massive with a mass $\mathrm{m}_{\tilde{A}}^{2}=\mathrm{m}_{\rho}^{2}$ and the remaining vectors $V_{\mu}$ of $S U(5)$ together with Majorana fermions $\lambda_{R}^{i} j$ that are the gauginos of unbroken $S U(5)$ symmetry stay massless. The righthanded components of the gaugino $\tilde{\lambda}_{R}$ combine with the left-handed chiral fermions $\varphi_{L}$ to become massive Dirac fermions with the same mass as the gauge boson $\tilde{A}$ : $\mathrm{m}_{\tilde{A}}^{2}=\mathrm{m}_{\rho}^{2}=\mathrm{m}_{\Psi}^{2}=40 g_{1}^{2} v^{2}$. This establishes the presence of a massive vector supermultiplet.

We end this part of the letter by remarking that one can also consider gauging either the $U(1)\left(g_{5}=0\right)$ or $S U(5)\left(g_{1}=0\right)$ symmetry. In the first case when gauging only the $U(1)$ symmetry, the minimum potential is at the same point as in the $S U(5) \times U(1)$ gauging. Therefore the above discussion applies here and one gets the same spectrum with equal masses for the $U(1)$ gauge multiplet. On the other hand, if only $S U(5)$ is gauged, the potential reaches its minimum at $z=k=0$. Then no supersymmetry breaking or internal symmetry breaking occurs and all particles in the theory are massless.

We now turn our attention to another well known model with a phenomenologically interesting particle spectrum, defined on the homogeneous coset space $E_{6} / S O(10) \times U(1)$ [13, 14]. The target manifold $E_{6} / S O(10) \times U(1)$ is parametrized by 16 complex fields $z^{\alpha}$ in a chiral superfield $\Phi_{\alpha}=\left(z_{\alpha}, \psi_{L \alpha}, H_{\alpha}\right)(\alpha=$ $1, \ldots, 16)$, transforming as a Weyl spinor under $S O(10)$.

\footnotetext{
2 No subtleties occur in this case, since the model has a nonsingular vacuum.
} 
Their chiral fermion superpartners have the quantum numbers of one full generation of quarks and leptons, including a right-handed neutrino. To cancel the $U(1)$ anomaly the model is extended to a complete 27 of $E_{6}$. According to the branching rule: $\underline{27} \rightarrow \underline{16}(1)+\underline{10}(-2)+$ $\underline{1}(4)$, where the numbers in parentheses denote the relative $U(1)$ weights. With this choice of matter content, the cancellation of chiral anomalies of the full $E_{6}$ isometry group is achieved 12] by introducing a superfield $\Psi_{m}=\left(N_{m}, \chi_{L m}\right)(m=1, \ldots, 10)$ which is equivalent to a $\underline{10}$ of $S O(10)$ with $U(1)$ charge -2 ; and finally a singlet $\Lambda=\left(h, \chi_{L}\right)$ of $S O(10)$, with $U(1)$ charge +4 .

The anomaly-free supersymmetric $\sigma$-model on $E_{6} /[S O(10) \times U(1)]$, is then defined by three chiral superfields $\left(\Phi_{\alpha}, \Psi_{m}, \Lambda\right)$ with Kähler potential given by

$$
\begin{aligned}
\mathcal{K}(\Phi, \bar{\Phi} ; \Psi, \bar{\Psi} ; \Lambda, \bar{\Lambda})= & K_{\sigma}+e^{-6 f^{2} K_{\sigma}}|h|^{2} \\
& +g_{m n} \bar{N}_{m} N_{n} e^{6 f^{2} K_{\sigma}},
\end{aligned}
$$

with $K_{\sigma}=\bar{z} \cdot\left[Q^{-1} \ln (1+Q)\right] . z$, the $\sigma$-model Kähler potential. We have introduced a constant $f$ with the dimension $m^{-1}$, determining the scale of symmetry breaking $E_{6} \rightarrow S O(10) \times U(1)$. The positive definite matrix $Q$ is defined as

$$
\begin{aligned}
Q_{\alpha}^{\beta} & =\frac{f^{2}}{4} M_{\alpha \gamma}^{\beta \delta} \bar{z}^{\gamma} z_{\delta}, \\
M_{\alpha \gamma}^{\beta \delta} & =3 \delta_{\alpha}^{+\beta} \delta_{\gamma}^{+\delta}-\frac{1}{2} \Gamma_{m n \alpha}^{+\beta} \Gamma_{m n \gamma}^{+} \delta
\end{aligned}
$$

Here $\Gamma_{m n}^{+}=\Gamma_{m n} \delta^{+}$are the generators of the $S O(10)$ on positive chirality spinors of $S O(10)$ [13], and $\delta^{+}$is the $10-\mathrm{D}$ positive chirality projection operator. Furthermore $g_{m n}$ is the induced metric for the 10 -vector representation defined by

$$
g_{m n}=\frac{1}{16} \operatorname{tr}\left(g_{T}\left(\Sigma_{m} C\right)^{\dagger} g_{T}\left(\Sigma_{n} C\right)\right),
$$

with $g_{T}=\left(\mathbb{1}_{16}+Q\right)^{-2}$. The lagrangian constructed from the Kähler potential (20) is invariant under a set of holomorphic Killing vectors generating a non-linear representation of $E_{6}$ :

$$
\begin{aligned}
\delta z_{\alpha}= & \frac{i}{2} \theta \sqrt{3} z_{\alpha}-\frac{1}{4} \omega_{m n}\left(\Gamma_{m n}^{+} \cdot z\right)_{\alpha}+\frac{1}{2}\left[\frac{i}{f} \epsilon_{\beta} \delta_{\alpha}^{\beta}\right. \\
& \left.-\frac{i f}{4} \bar{\epsilon}^{\beta} M_{\alpha \beta}^{\gamma \delta} z_{\gamma} z_{\delta}\right], \\
\delta h= & 2 i(\sqrt{3} \theta-3 f \bar{\epsilon} \cdot z) h, \\
\delta N_{n}= & -i \sqrt{3} \theta N_{n}-\omega_{n m} N_{m}-i f \bar{\epsilon} \cdot\left(\Gamma_{m n}^{+}\right. \\
& \left.-3 \delta_{m n}^{+}\right) \cdot z N_{m} .
\end{aligned}
$$

where $\delta_{m n}^{+}=\delta_{m n} \delta^{+}$, and $\theta, \omega_{m n}, \epsilon_{\alpha}$ and $\bar{\epsilon}^{\alpha}$ are the infinitesimal parameters of the $U(1), S O(10)$ and broken $E_{6}$ generators respectively. The corresponding Killing potentials are

$$
\begin{aligned}
\mathcal{M}_{i}= & M_{i} E \\
& -\frac{1}{8} e^{6 f^{2} K_{\sigma}} M_{i, \alpha}^{\beta} g_{T \gamma}^{\delta}\left(C \bar{\Sigma}_{m}\right)^{\alpha \gamma}\left(\Sigma_{n} C\right)_{\beta \delta} \bar{N}_{m} N_{n},
\end{aligned}
$$

with $E$ and the $\sigma$-model Killing potentials $M_{i}=$ $\left(M_{\theta}, M^{(m n)}, \bar{M}^{\beta}, M_{\beta}\right)$ given by

$$
\begin{aligned}
M_{\theta} & =\frac{1}{f^{2} \sqrt{3}}-\frac{1}{2} \sqrt{3} \bar{z}^{\alpha} K_{\sigma, \alpha} \quad \bar{M}^{\beta}=-\frac{1}{f} K_{\sigma,}{ }^{\beta}, \\
M^{m n} & =-\frac{i}{2} \bar{z}^{\alpha} \Gamma_{m n \alpha}^{+}{ }^{\beta} K_{\sigma, \gamma}, \quad M_{\beta}=-\frac{1}{f} K_{\sigma, \beta}, \\
E & =1-6 f^{2} e^{-6 f^{2} K_{\sigma}}|h|^{2}+6 f^{2} e^{6 f^{2} K_{\sigma}} g_{m n} \bar{N}_{m} N_{n} .
\end{aligned}
$$

Apart from the pure supersymmetric $\sigma$-model determined by this Kähler potential (20), we consider models in which (part of) the isometries (23) are gauged. As the $E_{6}$ is broken, the Higgs mechanism operates as follows: the Goldstone bosons $\left(\bar{z}^{\underline{\alpha}}, z^{\alpha}\right)$ are absorbed in the longitudinal component of the charged vector bosons, and if the full $E_{6}$ is gauged, we may choose the unitary gauge $\bar{z}^{\underline{\alpha}}=z_{\alpha}=0$. To analyze the model in this gauge, we introduce the covariant derivatives for the dynamical fields. The expressions for gauge-covariant derivatives of the complex scalar fields read

$$
\begin{aligned}
D_{\mu} z_{\alpha}= & \partial_{\mu} z_{\alpha}-g\left(\frac{i}{2} \sqrt{3} z_{\alpha} A_{\mu}+\frac{1}{4}\left(\Gamma_{m n}^{+} z\right)_{\alpha} A_{\mu(m n)}\right. \\
& \left.+\frac{1}{2}\left(\frac{i}{f} A_{\alpha \mu}-\frac{i f}{4} \bar{A}_{\mu}^{\beta} M_{\alpha \beta}^{\gamma \delta} z_{\gamma} z_{\delta}\right)\right) \\
D_{\mu} h= & \partial_{\mu} h-2 i g\left(\sqrt{3} A_{\mu}-3 f \bar{A}_{\mu}^{\alpha} z_{\alpha}\right) h \\
D_{\mu} N_{n}= & \partial_{\mu} N_{n}+i \sqrt{3} g A_{\mu} N_{n}+g A_{\mu(m n)} N_{m} \\
& +i f g \bar{A}_{\mu} \cdot\left(\Gamma_{m n}^{+}-3 \delta_{m n}^{+}\right) \cdot z N_{m}
\end{aligned}
$$

Here we have introduced the notation $\left(A_{\mu \alpha}, \bar{A}_{\mu}^{\alpha}\right)$ for the 32 charged gauge fields corresponding to the broken $E_{6}$ transformations; $A_{\mu(m n)}$ and $A_{\mu}$ are the gauge fields for the remaining $S O(10)$ and $U(1)$ transformations respectively.

Next we discuss in some detail the gauging of the full non-linear $E_{6}$ and the resulting particle spectrum of the theory. We choose to study the model in the unitary gauge in which all the Goldstone bosons vanish: $z^{\alpha}=\bar{z}_{\alpha}=0$. This implies that the broken Killing potentials $\overline{\mathcal{M}}^{\beta}$ and $\mathcal{M}_{\beta}$ vanish automatically, leaving us with $S O(10)$ and $U(1)$ Killing potentials $\mathcal{M}_{\theta}$ and $\mathcal{M}_{m n}$; and the $D$-term potential reads

$$
V_{D}=\frac{g^{2}}{2}\left(\mathcal{M}_{\theta}^{2}+\frac{1}{2} \mathcal{M}_{m n}^{2}\right)
$$

In the unitary gauge the potential becomes

$$
V_{\text {unitary }}=\frac{g^{2}}{2}\left(\frac{1}{f^{2} \sqrt{3}}-2 \sqrt{3}|h|^{2}+\sqrt{3} \sum_{m}\left|N_{m}\right|^{2}\right)^{2}
$$




$$
+\frac{g^{2}}{2} \sum_{m, n}\left|\bar{N}_{m} N_{n}-\bar{N}_{n} N_{m}\right|^{2} .
$$

Minimization of the potential leads to the following set of supersymmetric minima characterized by the equation

$$
\left|\bar{N}_{m} N_{n}-\bar{N}_{n} N_{m}\right|^{2}=0, \quad|h|^{2}=\frac{1}{6 f^{2}}+\frac{1}{2} \sum_{m}\left|N_{m}\right|^{2} .
$$

The value of the potential vanishes: $\langle V\rangle=0$, hence it is the absolute minimum of the potential. From (29), it follows that $|h| \neq 0$ and the $U(1)$ gauge symmetry is always broken; a solution with $\left|N_{m}\right|=0$ is possible, preserving $S O(10)$. However, this solution is not acceptable by itself, as it leads to the to the vanishing of the metric of the $\sigma$-model fields

$$
\begin{aligned}
\left\langle G_{\alpha}{ }^{\beta}\right\rangle & =\delta_{\alpha}^{\beta}\left(\frac{1}{f^{2}}-6|h|^{2}+18\left|N_{m}\right|^{2}\right)-4 \bar{N}_{m} N_{n}\left(\Gamma_{m n}^{+}\right)_{\alpha}^{\beta} \\
& =0 .
\end{aligned}
$$

Hence the masses of the $32 E_{6}$ gauge fields $A_{\mu}^{\alpha}$ vanish, and the four-fermion term $R_{z^{\alpha} \bar{z}^{\beta} h \bar{h}} \bar{\psi}_{R}^{\alpha} \psi_{L}^{\frac{\beta}{\alpha}} \bar{\chi}_{L} \chi_{R}$ diverges, just like in the $S O(10) / U(5)$-spinor model. Clearly, in this domain the model no longer correctly describes the physics of the situation (i.e., the correct vacuum and the corresponding spectrum of small fluctuations). Therefore we add soft breaking mass terms for the singlet $h$ and the vector $N_{m}$ :

$$
V_{\text {soft }}=\mu_{1}^{2} e^{-6 K_{\sigma}}|h|^{2}+\mu_{2}^{2} g_{m n} \bar{N}_{m} N_{n} e^{6 f^{2} K_{\sigma}},
$$

to shift the minimum a way from the singular point. The full scalar potential with soft breaking term in the unitary gauge is then:

$$
V=V_{\text {unitary }}+\mu_{1}^{2}|h|^{2}+\mu_{2}^{2}\left|N_{m}\right|^{2} .
$$

We now determine the mass spectrum of the theory. As the complex scalar transforms only under $U(1)$, we choose the unitary gauge for the $U(1)$ symmetry, which allow us to write

$$
h=\left(v+\frac{1}{\sqrt{2}} \rho\right) e^{\frac{1}{\sqrt{2 v}} i \kappa},
$$

where $\kappa$ is the longitudinal component of the massive gauge field $A_{\mu}$. Expanding the potential (32) to second order in $\rho$ and in the complex fluctuations $\tilde{N}_{m}$ around the minimum

$$
\left|N_{m}\right|^{2}=0, \quad|h|^{2}=v^{2}=\frac{1}{6 f^{2}}-\frac{\mu_{1}^{2}}{12 g^{2}} \quad \mu_{1}^{2}<2 \frac{g^{2}}{f^{2}}
$$

one obtains the following bosonic mass spectrum:

$$
\begin{gathered}
\mathrm{m}_{\tilde{A}}^{2}=\mathrm{m}_{\rho}^{2}=24 g^{2} v^{2}, \quad \mathrm{~m}_{A^{\alpha}}^{2}=\frac{\mu_{1}^{2}}{4 f^{2}}, \\
\mathrm{~m}_{\tilde{N}_{m}}^{2}=\frac{1}{f^{2}}\left(\frac{1}{2} \mu_{1}^{2}+\mu_{2}^{2}\right), \quad \mathrm{m}_{A_{n m}}^{2}=0 .
\end{gathered}
$$

As expected the gauge bosons $A_{\mu[m n]}$ of the non-broken $S O(10)$ symmetry remain massless. Analyzing the kinetic and mass terms of the fermions one realizes that two massive Dirac fermions can be formed by combining the fermions of the chiral multiplets with two gauginos:

$$
\Psi_{\alpha}=\frac{1}{\sqrt{2}} \lambda_{R \alpha}-i \sqrt{2} \frac{\mu_{1}}{2 g} \psi_{L \alpha}, \quad \Omega=\lambda_{R}-i \chi_{L} .
$$

The masses of these spinors read: $\mathrm{m}_{\Psi}^{2}=\frac{\mu_{1}^{2}}{2 f^{2}}$ and $\mathrm{m}_{\Omega}^{2}=$ $24 g^{2} v^{2}$. The $\underline{16}$ of the left-handed gaugino's $\lambda_{L \alpha}$ and quasi-Goldstone fermions $\chi_{L n}$ remain massless, together with the Majorana fermions $\lambda^{m n}$ that are gauginos of the unbroken $S O(10)$ symmetry. Therefore, in this model the gaugino components $\lambda_{L \alpha}$ are now to be identified with a family of quarks and leptons, rather than the quasi Goldstone fermions themselves. (We have observed a similar thing to happen also in the $S O(10) / U(5)$-spinor model discussed earlier.)

The gauging of the $S O(10) \times U(1)$ symmetry instead of the full $E_{6}$ gives analogous, but not quite identical, results. Also in this case one finds the potential (27), but in general with different values $g_{1}$ and $g_{10}$ for the coupling constants of $S O(10)$ and $U(1)$. Except for special values of the parameters, it has a minimum for the $S O(10)$ invariant solution, with $\left\langle z_{\alpha}\right\rangle=0$; and again the metric becomes singular. One way to shift the minimum away from this point is by introducing soft breaking terms (31). Another option is to add an extra Fayet-Iliopoulos term as the gauge group possesses an explicit $U(1)$ factor. In the first case, one obtains similar mass spectra as in the model with fully gauged $E_{6}$, but with $g=g_{1}$ and only one massive Dirac fermion $\Omega$ defined in (36). The gaug$\operatorname{inos} \lambda^{m n}$ that are left over remain unpaired, and hence massless. Furthermore, the chiral fermions $\psi_{L}^{\alpha}$ and $\chi_{L}^{n}$ remain massless. In the second case, for special values of the coupling constants $g_{1}$ and $g_{10}$, or the Fayet-Iliopoulos parameter $\xi$, one can get different results. Since the $S O(10)$ and $U(1)$ coupling constants are independent, one may choose to gauge only $S O(10)\left(g_{1}=0\right)$. In that case both supersymmetry and internal symmetry are preserved, and the particle spectrum of a model contains of a massless $S O(10)$ gauge boson, just like in the usual supersymmetric $S O(10)$ grand unified models.

Solutions with $\left|N_{m}\right| \neq 0$ breaking $S O(10)$ are allowed, and expected in the next stage of the symmetry breaking. For example, $S O(10)$ broken solution can be chosen as

$$
\begin{aligned}
f \bar{N}_{m} & =\left(\begin{array}{llllllllll}
0 & 0 & 0 & 0 & 0 & 0 & 0 & 0 & 0 & v_{10}
\end{array}\right), \\
|h|^{2} & =\left|v_{h}\right|^{2}=\frac{1}{6 f^{2}}+\frac{v_{10}^{2}}{2 f^{2}} .
\end{aligned}
$$

Since the complex scalar $N_{m}$ gets a vacuum expectation value; this breaks the internal linear $S O(10)$ symmetry, leaving only $S O(9)$. As supersymmetry is preserved, one expects the spectrum of physical states to fall into supersymmetric multiplets with vanishing mass supertrace. 
Indeed the general mass sum rule ${ }^{3}[15,16]$ leads to

$$
\mathrm{S} \operatorname{Tr} \mathrm{m}^{2}=2 g^{2} G^{\underline{I}} \mathcal{M}_{i} \mathcal{M}_{i, \underline{I}}=0 .
$$

In this paper, I have discussed in some detail the analysis of of particle spectrum of supersymmetric $\sigma$-models on homogeneous coset-spaces $E_{6} /[S O(10) \times U(1)]$ and $S O(10) / U(5)$. I have analyzed the possible vacuum configurations of these models and investigate the existence of the zeros of the potential, for which the models are anomaly-free, with positive definite kinetic energy. The consequences of these physical requirements have been analyzed. We found that there exist supersymmetric minima for both these models when the full isometry groups $E_{6}$ and $S O(10)$ are gauged. The analysis is straightforward as one can employ the unitary gauge to put the Goldstone bosons to zero. In some cases, we find that the Kähler metrc is singular: the kinetic energy of the would-be Goldstone modes and their fermionic partners vanishes in the vacuum. We showed by addi-

3 As we have gauge the full $E_{6}$ the standard linear Fayet-Iliopoulos term is of course absent. tion of soft supersymmetry-breaking mass parameters, that the minimum can be shifted away from the singular point. The particle spectrum in the presence of soft supersymmetry-breaking mass parameters is computed.

Continuing our line of investigation of the particle spectrum of supersymmetric $\sigma$-models on $E_{6} /[S O(10) \times U(1)]$, and $S O(10) \times U(1)$, we have also studied the possibility of gauging (part of) the linear subgroups, i.e., $S O(10) \times U(1)$ and $U(5)$. In each of these models, we found that the properties of the model investigated depend to a certain extent on the value of parameters (gauge couplings, Fayet-Iliopoulos term.) We have obtained all supersymmetric minima, of which some are physically problematic as the kinetic terms of the Goldstone multiplets either vanish or have negative values.

I thank J.W. van Holten and J. Babington for very useful suggestions and for reading the manuscript.
[1] B. Zumino "Supersymmetry and Kähler manifolds" Phys. Lett. B87 (1979) 203.

[2] D. Freedman and L. Alvarez-Gaumé "Geometrical structure and ultraviolet finiteness in the supersymmetric sigma model" Commun. Math. Phys. 80 (1981) 433.

[3] S. Groot Nibbelink T.S. Nyawelo and J.W. van Holten "Construction and analysis of anomaly-free supersymmetric $S O(2 N) / U(N) \sigma$-models" Nucl. Phys. B 594 (2001) 441-476; hep-th/0008097.

[4] T.S. Nyawelo, F. Riccioni, S. Groot Nibbelink and J.W. van Holten "Singular supersymmetric sigma models" Nucl. Phys. B663 (2003) 60-78; hep-th/0302135.

[5] C-L. Ong "Gauged supersymmetric generalized nonlinear sigma models for quarks and leptons" Phys. Rev. D27 (1983) 3044.

[6] G. Moore and P. Nelson "Anomalies in nonlinear sigma models" Phys. Rev. Lett. 53 (1984), 1519.

[7] E. Cohen and C. Gomez "Dynamical implications of anomaly in nonlinear sigma models" Nucl. Phys. B254 (1985), 235.

[8] W. Buchmüller and W. Lerche "Geometry and anomaly structure of supersymmetric sigma models" Ann. Phys. 175 (1987), 159.

[9] A. C. W. Kotcheff, G. M. Shore "Kähler sigma models from supersymmetric gauge theories" Int. J. Mod. Phys. A4 (989) 4391.

[10] M. Bando, T. Kuramoto, T. Maskawa, and S. Uehara
"Structure of nonlinear realization in supersymmetric theories" Phys. Lett. B138 (1984) 94.

[11] M. Bando, T. Kuramoto, T. Maskawa, and S. Uehara "Nonlinear realization in supersymmetric theories" Prog. Theor. Phys. 72 (1984) 313.

[12] S. Groot Nibbelink and J.W. van Holten "Matter coupling and anomaly cancellation in supersymmetric $\sigma$-models" Phys. Lett. B442 (1998) 185-191; hep-th/9808147.

[13] Y. Achiman S. Aoyama and J.W. van Holten "Gauged supersymmetric sigma models and $E_{6} /[S O(10) \times U(1)]$ " Nucl. Phys. B285 (1985) 179.

[14] Y. Achiman S. Aoyama and J.W. van Holten "The nonlinear supersymmetric sigma models on $E_{6} /[S O(10) \times$ U(1)]" Phys. Lett. B141 (1984) 64.

[15] M. T. Grisaru, F. Riva and D. Zanon "The one loop effective potential in superspace" Nucl. Phys. B214 (1983) 465.

[16] S. J. Gates, M. T. Grisaru, M. Rocek and W. Siegel "Superspace or one thousand and one lessons in supersymmetry" Front. Phys. 58 (1983) 1-548; hep-th/0108200.

[17] J. Bagger and E. Witten "The gauge invariant supersymmetric nono-linear sigma model" Phys. Lett. B118 (1982) 103-106.

[18] J. Wess and J. Bagger, Supersymmetry and supergravity Princeton Series in Physics, Princeton University Press, 1992. 